\title{
Hacia la integración del conocimiento disciplinar y pedagógico: desarrollando el conocimiento pedagógico del contenido en la formación inicial de profesores de química*
}

\author{
Towards the integration of disciplinary and pedagogical knowledge: developing \\ pedagogical content knowledge in the training of pre-service chemistry teachers

\section{Ainoa Marzábal ${ }^{a}$, Patricia Moreira ${ }^{b}$, Virginia Delgado, Jeanette Moreno ${ }^{d}$, Raúl Contreras ${ }^{e}$} \\ a Pontificia Universidad Católica de Chile. Correo electrónico: amarzabal@uc.cl \\ b Pontificia Universidad Católica de Chile. Correo electrónico: pmmoreira@uc.cl \\ c Pontificia Universidad Católica de Chile. Correo electrónico: vcdelgad@uc.cl \\ d Pontificia Universidad Católica de Chile. Correo electrónico: dmorenoc@uc.cl \\ e Pontificia Universidad Católica de Chile. Correo electrónico: rcontrer@uc.cl
}

\section{RESUMEN}

Como estrategia para promover la articulación entre el conocimiento disciplinar y pedagógico en la formación inicial de profesores de Química, se propone la noción de conocimiento pedagógico del contenido como modelo integrador basado en la reflexión didáctica y pedagógica en torno a los contenidos disciplinares. Para el diseño de cursos que generen y desarrollen el conocimiento pedagógico del contenido en la formación inicial de profesores especialistas, ha sido necesario consensuar una visión de qué es este tipo de conocimiento y de qué manera se puede promover, identificar conocimientos químicos clave para la enseñanza en educación media, proponer una secuencia para su desarrollo en la formación inicial e implementar estrategias específicas para elicitar este conocimiento profesional. En este trabajo presentamos el resultado de las discusiones que como equipo hemos desarrollado, y un ejemplo de las actividades propuestas y las producciones de nuestros profesores en formación.

Palabras clave: profesores de Química, formación inicial docente, conocimiento pedagógico del contenido, representaciones del contenido

\begin{abstract}
As a strategy to promote the articulation between disciplinary and pedagogical knowledge in the training of pre-service chemistry teachers, we propose the notion of pedagogical content knowledge as an integrative model based on the pedagogical reflection of the disciplinary content. In order to design courses that create and develop pedagogical content knowledge in the training of specialist teachers, it was necessary to agree on a vision of what kind of knowledge this is and how it can be promoted, as well as, to identify the main chemical knowledge for teaching in secondary education, to propose a development sequence for initial training, and to implement specific strategies to elicit this professional knowledge. This paper presents the results of the discussions that have been developed as a team, an example of the proposed activities and the productions of our teachers in training.

Key words: chemistry teachers, pre-service teachers' training, pedagogical content knowledge, content representations.
\end{abstract}

\footnotetext{
Producto del proyecto de investigación en docencia universitaria "Exploración de la transformación del conocimiento químico para su enseñanza, a lo largo del curso Diseño de laboratorio" (PUC1201).
} 
Estudios Pedagógicos XLII, N 4: 243-260, 2016

HACIA LA INTEGRACIÓN DEL CONOCIMIENTO DISCIPLINAR Y PEDAGÓGICO: DESARROLLANDO EL

CONOCIMIENTO PEDAGÓGICO DEL CONTENIDO EN LA FORMACIÓN INICIAL DE PROFESORES DE QUÍMICA

\section{LA FORMACIÓN DE LOS PROFESORES DE CIENCIAS EXPERIMENTALES}

Desde los años 80 la investigación en Didáctica de las Ciencias ha dirigido su atención hacia la formación de profesores de ciencias, dada su relevancia en los procesos de enseñanza y aprendizaje (Barber \& Mourshed, 2007). En el caso de la formación inicial, se cuestionan los planteamientos que visualizan la formación del profesorado como una yuxtaposición de saberes académicos que hay que aplicar en la práctica, considerando que pueden integrarse solucionando una de las asignaturas pendientes de la formación inicial: la integración del conocimiento disciplinar y pedagógico (Furió, 1994).

Actualmente en Chile existen dos posibilidades de formación de profesores de ciencias: formación paralela, en la que se obtienen conocimientos de la especialidad y de pedagogía en forma paralela; y consecutiva, en la que se obtienen primero los conocimientos de la especialidad, y después los conocimientos y experiencia pedagógica (Cofré \& Vergara, 2010).

Los currícula de formación en paralelo, que son los más frecuentes, consideran cursos de ciencias y de educación. En los cursos de ciencias suele predominar la extensión sobre la profundidad en el contenido, las clases son expositivas, se estandarizan los procedimientos y se usa material sofisticado en las prácticas de laboratorio; y los cursos de educación están descontextualizados de los contenidos científicos que los profesores van a tener que enseñar. Este tipo de modelo paralelo, denominado consecutivo o no integrado, que se ha caracterizado por una formación disciplinar y pedagógica sin una articulación explícita, es hoy en día el más común, a pesar de las evidencias que sugieren que este tipo de formación no da lugar a la configuración identitaria del profesorado necesaria para ejercer su rol docente, lo que suele derivar en una crisis en su inserción profesional (Bolivar, 2007).

Por el contrario, los modelos paralelos simultáneos, en los que la formación disciplinar y pedagógica está articulada, parecen producir una identidad profesional más adecuada a la realidad, en la que el futuro profesor recibe una formación específica y práctica en aquellos aspectos en los que se juega el éxito o el fracaso de la enseñanza (Esteve, 2003).

El análisis de los programas de formación inicial de profesores de ciencias evidencia que mundialmente existe un fuerte movimiento de cambio y transformación en esta dirección, que lleva a resignificar la formación de los profesores de ciencias, orientándola hacia el desarrollo de un profesional autónomo, reflexivo e investigador de su propio quehacer (Camacho et al., 2010).

Cofré y sus colaboradores han realizado un análisis exhaustivo de la formación inicial de profesores de ciencias en Chile, encontrando que las trayectorias curriculares son mayoritariamente paralelas, con una gran heterogeneidad entre los modelos formativos, con distinta duración y modalidad de especialización (Cofré et al., 2010). En todos los casos, la formación de profesores tiene un fuerte componente disciplinar y pedagógico, y poco énfasis en la formación práctica, de investigación y didáctica de las ciencias (Carrascosa et al., 2008), concluyendo que es imprescindible que se empiecen a incluir cursos explícitos de temas tan importantes como la investigación en ciencias y en didáctica, así como actividades curriculares que incluyan la historia y la naturaleza de la ciencia. 


\section{ANTE UN NUEVO PARADIGMA EN LA FORMACIÓN INICIAL DE PROFESORES DE QUÍMICA}

A partir del año 2012, la formación de profesores en Chile empieza un proceso de transformación activado por los Convenios de Desempeño en el ámbito de la formación inicial docente, un instrumento competitivo de adjudicación de recursos de alto impacto estratégico que provee de financiamiento en base a resultados y permite a la institución generar iniciativas transformadoras focalizadas en aspectos asociados al mejoramiento de la calidad de la formación de profesores (Mineduc, 2012).

En la primera convocatoria, la Pontificia Universidad Católica de Chile se adjudica un convenio de desempeño centrado en la "integración de competencias disciplinares, pedagógicas y profesionales" para mejorar la efectividad de los procesos de enseñanza y aprendizaje, respondiendo a las problemáticas en la formación de profesores que han sido identificadas tanto a nivel internacional como nacional. Ante este reto, se opta por un modelo paralelo simultáneo, donde los saberes disciplinares y pedagógicos se puedan integrar.

Ante el reto de lograr esta integración de saberes, se considera la revisión bibliográfica de Chung Wei y sus colaboradores (2010), que concluyen que:

a) El impacto del desarrollo profesional durante la formación es relevante y aporta al incremento de logros de aprendizaje;

b) El contenido del desarrollo profesional aporta más al focalizarse en cómo utilizar destrezas pedagógicas y cómo enseñar contenidos específicos a los alumnos que en discusiones abstractas sobre educación;

c) Al comparar el efecto de diversas orientaciones formativas en el aprendizaje de los alumnos, los mayores logros se obtienen cuando los profesores han participado de una formación focalizada en la profundización de su conocimiento disciplinario integrado a la práctica de la enseñanza en un ambiente de continua colaboración profesional.

El equipo de la PUC introduce el concepto de Conocimiento Pedagógico del Contenido (CPC), como articulador de los saberes pedagógicos y disciplinares. La noción de CPC trata de superar la dicotomía entre el conocimiento pedagógico y disciplinar (Schmelzing, et al., 2013), y fue acuñada en 1987 por Lee Shulman como parte del conocimiento profesional del profesor, en lo que denominó el paradigma perdido de la enseñanza. Se trata del constructo teórico más ampliamente utilizado en la última década para la formación de profesores (Loughran et al., 2012), si bien en Chile parecería tratarse de un paradigma perdido, al menos hasta ahora, ya que son escasas las oportunidades que poseen los futuros docentes de desarrollar este conocimiento durante su formación, al no estar explícitas en el currículum (Vergara y Cofré, 2014).

Para Shulman (2005), un profesor sabe algo que otros -los alumnos- no comprenden. El profesor requiere formas de expresar, exponer, escenificar o representar de otra manera ideas, de tal manera que los que no saben puedan llegar a saber, los que no entienden puedan comprender y discernir, y los inexpertos puedan convertirse en expertos. Desde esta perspectiva, la enseñanza se inicia en el proceso mediante el cual el profesor selecciona un conjunto de representaciones y acciones pedagógicas a partir de la comprensión de lo que se ha de aprender, y cómo se lo debe enseñar a los estudiantes. 
Shulman organiza el conocimiento profesional del profesor en torno a siete dimensiones: conocimiento del contenido, conocimiento didáctico general, conocimiento del curriculum, conocimiento pedagógico del contenido, conocimiento del alumnado, conocimiento del contexto escolar y conocimiento de las finalidades educativas. Para el autor, el CPC adquiere particular interés porque identifica los cuerpos de conocimientos distintivos para la enseñanza. El CPC representa la mezcla entre materia y didáctica, por lo que se llega a una comprensión de cómo determinados temas y problemas se organizan, se representan y se adaptan a los diversos intereses y capacidades de los alumnos, y se exponen para su enseñanza (Shulman, 2005).

En el ámbito de la educación científica, y en particular de la didáctica de la Química, los resultados de la investigación han comenzado a borrar la frontera entre conocimiento químico y pedagógico: hoy contamos con información específica sobre las ideas previas, teorías intuitivas y dificultades conceptuales de los alumnos, y tenemos una idea más clara de su influencia sobre el aprendizaje (Kind, 2009). Resultados como estos hacen pensar que la habilidad de un docente para crear condiciones que faciliten el aprendizaje no sólo depende de sus conocimientos sobre el tema o sobre variados métodos de enseñanza, sino más bien de su habilidad para transformar el conocimiento disciplinar en formas que resulten significativas para los estudiantes (Talanquer, 2004). Esta transformación pedagógica del conocimiento químico requiere que el docente domine la materia, pero con el propósito de enseñarla. El tipo de conocimiento que permite esta recreación del contenido, el CPC, está compuesto por una amalgama entre conocimiento disciplinar, didáctico y pedagógico, que requiere que el profesor integre estas tres dimensiones en una estructura coherente que guíe los pensamientos, decisiones y acciones en el aula. Y es el tipo de conocimiento que permitiría al profesor satisfacer las demandas profesionales que deberá enfrentar en el sistema escolar: identificar las ideas, conceptos y preguntas centrales asociadas con un tema; reconocer las posibles dificultades de los estudiantes, y cómo impactan en el aprendizaje; identificar preguntas, problemas o actividades que obliguen al estudiante a reconocer y cuestionar sus ideas previas; seleccionar experimentos, problemas o proyectos, que permitan que los estudiantes exploren conceptos e ideas centrales en la disciplina; construir explicaciones, analogías o ejemplos, que faciliten la comprensión de los conceptos abstractos; o diseñar actividades de evaluación que permitan la aplicación de lo aprendido en contextos realistas y variados (Talanquer, 2004).

Si bien el CPC como dimensión clave de la formación profesional constituye una idea muy prometedora, su caracterización no ha sido fácil, porque la comunidad de investigadores en el ámbito de la formación de profesores todavía no ha generado un modelo consistente de CPC, ni tampoco existe consenso sobre cómo caracterizar este conocimiento en los profesores (Park \& Oliver, 2008). Hay quienes consideran que el CPC no es un tipo especial de conocimiento, sino el resultado de la aplicación del conocimiento didáctico y pedagógico a una disciplina particular -modelo integrado-; y aún cuando pueda ser considerado como conocimiento independiente, su desarrollo ocurre principalmente a través de la experiencia y la práctica en el aula. En el otro extremo están quienes sugieren que el CPC debería ser el eje central del diseño curricular de los programas de formación de profesores, alrededor de cursos integradores que promuevan el análisis, la discusión y la reflexión del contenido científico desde las perspectivas didáctica y pedagógica -modelo de transformación o complementario (Borowski et al., 2012). Nuestra propuesta se sitúa en un punto intermedio: creemos que el CPC es un tipo especial de conocimiento, que 
puede desarrollarse en múltiples instancias -entre las cuales la experiencia de aula será clave- y que se va construyendo en la medida en que se van integrando otros tipos de conocimiento docente: disciplinar, curricular, pedagógico, didáctico, contextual, entre otros. Sin embargo, no podemos pensar en el CPC como una superposición de ideas: las relaciones que se establecen entre las ideas que incorpora el futuro profesor como parte de su formación, junto con sus concepciones y creencias, dan lugar a nuevo conocimiento que se va construyendo a partir de instancias de cuestionamiento y estructuración de las ideas.

La integración de estos conocimientos profesionales no se da de manera natural y requiere de un nivel alto de reflexión. Así entonces, la formación inicial podría ser un buen espacio para acompañar la construcción del CPC, como la integración del conocimiento que los futuros docentes han adquirido en forma independiente, en una estructura coherente a la que posteriormente podrán incorporar sus experiencias como docentes. Este espacio debe crear las condiciones para cuestionar las creencias y el pensamiento docente espontáneo de los futuros docentes (Furió, 1995), y ofrecer múltiples oportunidades de práctica en el aula y de reflexión crítica sobre el trabajo desarrollado, que podría lograrse en los cursos terminales de la formación inicial.

Para orientar el desarrollo del CPC en la formación inicial de profesores, tomamos como supuesto la naturaleza dinámica del $\mathrm{CPC}$, que se genera y desarrolla continuamente desde la formación disciplinaria, la observación de clases, los cursos específicos de la enseñanza de la materia y la experiencia de enseñanza (Abell, 2007, 2008). Estos procesos no pueden estar desconectados, sino que las piezas clave del contenido a enseñar deben ser sujeto de análisis, y discusión didáctica y pedagógica. Para ello se requiere contar con formadores de formadores que tengan una adecuada preparación y experiencia práctica (Avalos, 2005), y que estén dispuestos a hacer visible su forma de pensar en química con el propósito de enseñarla. En el caso de la PUC, se pensó en la constitución de equipos interdisciplinarios de químicos y didactas que pudieran llevar a cabo esta tarea, considerando que su conocimiento puede ser complementario para acompañar las reflexiones de los futuros profesores en torno a las temáticas que deberán enseñar.

\section{DISEÑO DE UNA ESTRUCTURA DE LA UNIDAD PARA EL DESARROLLO DEL CPC EN QUÍMICA}

Una vez consensuada la visión del CPC en Química, y de su desarrollo en la formación inicial de profesores, empiezan a surgir las primeras ideas de las actividades que podrían permitir estos procesos de reflexión. El análisis de textos escolares en busca de ideas centrales, conceptos integradores y buenas actividades, el desarrollo y análisis de diversas formas de representar el contenido, la identificación de las ideas centrales de las temáticas que se pueden trabajar, el análisis de situaciones de aula o de producciones de los estudiantes, o el diseño de experimentos, problemas, trabajos o instrumentos de evaluación, nos parecieron buenas instancias para ello. Este tipo de reflexión no solo ayudaría a los profesores en formación a desarrollar su CPC, sino también la capacidad crítica y las habilidades analíticas que les permitirían concebir el aula como un espacio de exploración e investigación continua (Talanquer, 2004).

Esta primera etapa de diseño culmina con tres consideraciones que definirán la estructura de los cursos a diseñar: priorizar la profundidad por sobre la cobertura, intencionar una 
secuencia de aprendizaje para el desarrollo del CPC e implementar estrategias específicas para caracterizar el desarrollo del CPC de los profesores en formación.

\subsection{PRIORIZAR LA PROFUNDIDAD POR SOBRE LA COBERTURA}

Desde una perspectiva de promover la autonomía en el futuro profesor, se espera acompañar los procesos de reflexión sobre el contenido, para su enseñanza en algunas temáticas, de tal forma que se desarrollen un conjunto de competencias profesionales que posteriormente se podrán transferir a otras temáticas. De este modo, en los cursos no se pretende cubrir todas las temáticas del curriculum escolar chileno de Química, sino seleccionar aquellas que se consideren relevantes para modelar el proceso de transformar el conocimiento disciplinar.

Para la selección de temáticas se realiza un análisis de la malla curricular de formación disciplinar de los futuros profesores, de las temáticas cuyo aprendizaje ha sido identificado en la literatura didáctica como desafiante, de los estándares de formación de profesores y del curriculum chileno de Química (Mineduc, 2012). Se trata de aquellos tópicos específicos que son clave para el curriculum escolar, y que es vital que los profesores novatos tengan herramientas para enseñar (Bal \& Forzani, 2009).

Mediante este proceso se identifican las siguientes temáticas como buenas oportunidades para la reflexión didáctica y pedagógica sobre el contenido:
a) Teoría corpuscular de la materia
b) Propiedades periódicas y su relación con la reactividad
c) Termoquímica / Termodinámica
d) Cinética y equilibrio químico
e) Energía nuclear

\subsection{INTENCIONAR UNA SECUENCIA DE APRENDIZAJE}

Ante la diversidad de actividades que pueden promover la reflexión didáctica sobre el contenido, se evidencia la necesidad de organizar los procesos de reflexión con una intencionalidad didáctica.

En primer lugar, consideramos los sub-componentes del CPC que han sido identificados (Lee \& Luft, 2008): conocimiento sobre las estrategias de enseñanza del contenido específico, conocimiento de la comprensión que tienen los estudiantes del contenido, conocimiento de las formas de evaluar el contenido, y conocimiento de las metas y objetivos para la enseñanza del contenido en el plan de estudios.

En base a estos elementos, y la progresión propuesta por Shulman (2005) para el desarrollo del Conocimiento Pedagógico del Contenido, proponemos una estrategia de progresión que responde al modelo didáctico de razonamiento y acción (Acevedo, 2009) que se representa en la figura 1 y se desarrolla a continuación: 
Fig. 1: estrategia de progresión para el desarrollo del CPC en la formación inicial

$$
\text { Indagación en el contenido (problema químico) }
$$

Preparación y representación del contenido

Selección y adaptación del contenido (problema didáctico)

La transformación del conocimiento en términos de su enseñabilidad se inicia con la movilización del conocimiento químico para la resolución de un problema químico con una estrategia de indagación. Proponemos esta estrategia, considerando que, en los últimos veinte años, los organismos a cargo de diseñar la educación científica en diversos países han propuesto nuevos currícula cuyo propósito común ha sido promover que los docentes usen la indagación como enfoque de enseñanza (Minner et al., 2009), y es necesaria una formación específica para poder desarrollarla (Harlen, 2010).

Una vez que el futuro profesor activa su conocimiento disciplinar, se inicia la transformación mediante la preparación del contenido para su enseñanza. La preparación involucra el análisis de la enseñanza considerando interpretación y análisis crítico de textos escolares, estructuración y segmentación de las ideas, análisis curricular y clarificación de los objetivos. A continuación, se representa el contenido mediante un repertorio de representaciones que incluyen analogías, metáforas, ejemplos, demostraciones y/o explicaciones sobre el contenido. En este contexto los futuros docentes pueden desarrollar habilidades que les permitan reconocer, evaluar y construir diferentes representaciones de una misma idea o concepto con el fin de crear oportunidades de aprendizaje para todos los estudiantes.

Consideramos que las diferentes representaciones del contenido constituyen un repertorio didáctico, entre el cual el futuro profesor debe seleccionar y adaptar, de acuerdo a las características de los estudiantes y al contexto en el cual se encuentran, considerando características como sus ideas iniciales, dificultades y motivación. El problema didáctico constituye un contexto que, a modo de estudio de caso, proporciona un escenario que promueve la toma de decisiones del futuro profesor mediante la selección de representaciones y su adaptación al contexto específico.

\subsection{IMPLEMENTAR ESTRATEGIAS ESPECÍFICAS PARA CARACTERIZAR EL DESARROLLO DEL CPC}

Al trabajar en la generación y desarrollo del CPC, se requieren estrategias para extraer este conocimiento profesional de los profesores en formación, para evaluar de qué forma se va modificando a lo largo de esta línea formativa. Esto es especialmente significativo considerando que extraer el CPC de un profesor es un proceso muy complejo, al tratarse de conocimiento implícitos que hay que hacer explícitos, y cuyo proceso de articulación suele requerir un amplio periodo de tiempo (Loughran et al., 2006). 
En las tres etapas se generan instancias de explicitación del contenido científico que van cambiando, respondiendo a los diferentes propósitos de las actividades propuestas: la indagación en el tema científico, la generación de representaciones de este contenido para su enseñanza, y la adaptación de una representación a un contexto de enseñanza concreto, con criterios disciplinares, didácticos y pedagógicos. Sin embargo, es importante considerar estrategias que se enfoquen en caracterizar el CPC de los profesores en formación.

Una de las propuestas más utilizadas con este fin es la de Loughran y sus colaboradores (2004). Su propuesta considera estrategias para el CPC declarativo, centrado en los conocimientos sobre didáctica que posee el profesor, y para el CPC procedimental, más ligado a la forma en que el conocimiento sobre didáctica del profesor se puede ver reflejado en su acción docente. Dado que nuestros cursos son de naturaleza teórica, nos centramos en el CPC declarativo, que de acuerdo a la propuesta de los autores puede ser caracterizado mediante los CoRes (Content Representations).

Los CoRes son una generalización de las respuestas del profesorado, expresadas en forma de proposiciones, acerca del modo en que enfocan la enseñanza de un tema y las razones respecto de cómo lo van a hacer y por qué lo van a hacer de este modo. Los CoRes nos permiten comprender las decisiones que los docentes toman en la acción docente, aunque con ciertas limitaciones.

La extracción de los CoRes se realiza mediante entrevistas semiestructuradas que se organizan en torno a las siguientes preguntas, para cada contenido específico (Garritz, 2007):

1. ¿Qué intentas que los estudiantes aprendan alrededor de esta idea?

2. ¿Por qué es importante que los estudiantes aprendan esta idea?

3. ¿Qué conocimiento tienen los estudiantes en torno a esta idea, y de qué manera afecta su aprendizaje?

4. ¿Qué factores pueden dificultar en el aprendizaje de esta idea?

\section{DESARROLLANDO EL CONOCIMIENTO PEDAGÓGICO DE LA CINÉTICA EN PROFESORES DE QUÍMICA EN FORMACIÓN}

A continuación presentamos un ejemplo de las actividades desarrolladas en los cursos de $\mathrm{CPC}$, que nos parece puede contribuir a visualizar más claramente el tipo de trabajo que estamos realizando, y el conocimiento profesional que están construyendo los futuros docentes a través de sus CoRes.

Hemos situado esta actividad en el estudio de la cinética química, debido a su importancia en la comprensión del proceso de reacción química. Se trata de una temática que los estudiantes identifican como de difícil comprensión, lo que se evidencia en las concepciones alternativas que presentan estudiantes de educación secundaria y universitaria (Cakmacki, 2010).

Las dificultades que los estudiantes presentan parecen estar relacionadas con su tendencia a restringir las explicaciones a los aspectos macroscópicos de los fenómenos cinéticos, lo que podría explicar también que no logren extraer el conocimiento científico expresado mediante modelos simbólicos y matemáticos. Es por ello que en la enseñanza se debería fomentar el uso de diversas representaciones. 
Considerando la relevancia de la cinética, es clave que los profesores sean capaces de considerar en su enseñanza no solamente los aspectos fundamentales sobre la cinética sino también sobre su aprendizaje.

\subsection{RESOLUCIÓN DE UN PROBLEMA CINÉTICO}

Se les presentan a los estudiantes de pedagogía tres problemas contextualizados con situaciones experimentales con distinto orden de reacción y con aplicación de técnicas experimentales distintas:

Fig. 2: Problemáticas químicas para la movilización del conocimiento sobre cinética
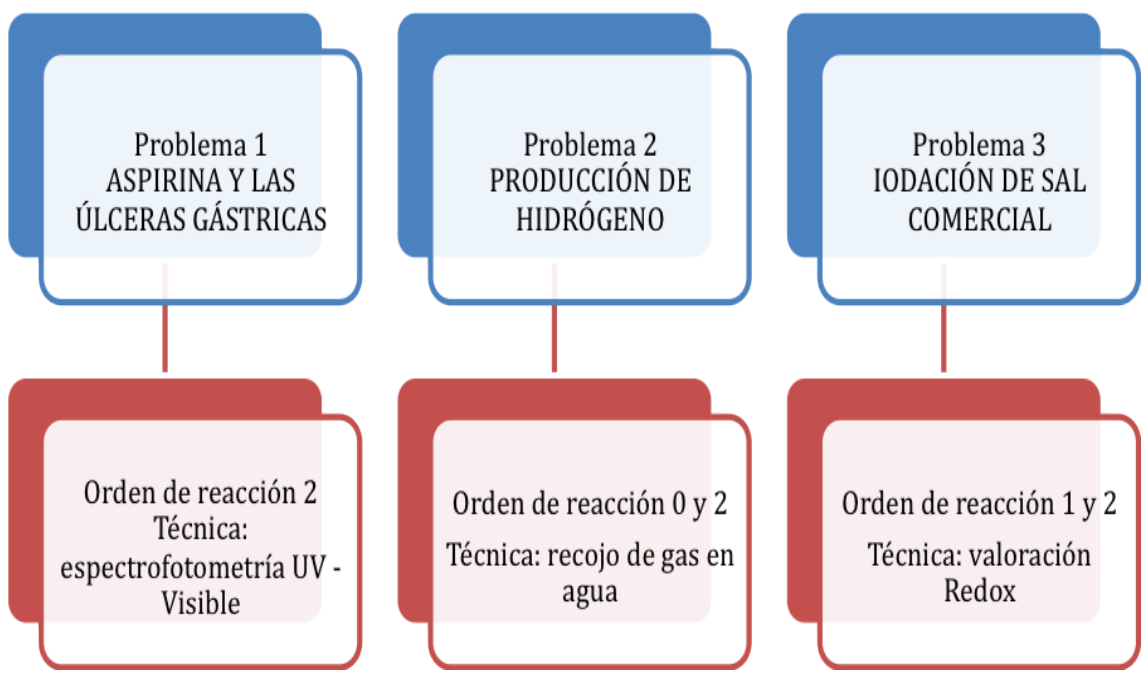

A continuación se detallan las situaciones problemáticas que se deberán resolver a partir de los diseños experimentales propuestos por cada estudiante:

Problema 1: Aspirina y las úlceras gástricas

"Para que un fármaco pueda ejercer su acción farmacológica sobre el funcionamiento de un determinado tejido, es necesario que alcance una concentración crítica en el medio que lo rodea, una concentración umbral. Así, la farmacocinética estudia los procesos que experimenta el fármaco en el organismo, desde su introducción hasta su eliminación. Aspirina es uno de los fármacos más utilizado en el mundo como antiinflamatorio y analgésico; éste fármaco puede ocasionar úlceras gástricas en el organismo es así que algunos médicos recomiendan una previa disolución en agua antes de su consumo, por lo cual se requiere a partir de un diseño experimental realizar un estudio cinético de disolución de tabletas de ácido acetilsalicílico "aspirina" en agua para evaluar el tiempo que demora en solubilizarse y dar las correctas indicaciones a los pacientes.” 
Problema 2: Producción de hidrógeno

"El hidrógeno es uno de los elementos con mayor importancia en nuestro día a día. Elevadas cantidades de hidrógeno son necesarias en industrias químicas y petrolíferas, como es el caso del proceso de "Haber" para la producción de amoníaco, el quinto compuesto con mayor producción industrial. Asimismo, el hidrógeno está actualmente siendo testeado como fuente de energía "limpia” para la utilización en transporte. Una de las formas más sencillas de obtener hidrógeno es la reacción de metales alcalinos con ácidos; tal es el caso de la reacción de magnesio metálico con ácido clorhídrico. Por lo tanto se requiere diseñar un protocolo experimental que nos permita realizar un estudio cinético del orden de reacción y así poder determinar la influencia de la concentración en la producción de Hidrógeno gas.”

Problema 3: Yodación de sal comercial

“La Organización Mundial de la Salud y el Fondo de las Naciones Unidas para la Niñez consideraron la yodación de la sal comestible como la medida preventiva de mayor cobertura, menos costosa y de gran eficacia en el mundo. El yodo una vez ingerido, es transformado en yoduro (la mayor parte) y luego es absorbido casi completamente en el intestino delgado. Por esta razón es que en las sales yodadas se prefiere la adición de yodo, sin embargo, en algunas sales comerciales es yoduro de potasio o sodio el adicionado, para facilitar su posterior adsorción; pero existe el problema de su oxidación ya sea enzimática o por otros agentes presentes en la alimentación, lo que dificultaría su posterior absorción. A raíz de esto y con el fin de evaluar el consumo de sal yodada se requiere realizar un estudio cinético para determinar el tiempo que el yoduro permanece como tal sin ser oxidado a yodo y considerando que hay presente sales minerales en el momento de la ingestión de la sal, evaluar la oxidación con y sin presencia de sulfato de cobre."

Las actividades propuestas fueron desarrolladas por tres estudiantes de $5^{\circ}$ semestre del programa de formación inicial de profesores de Química de la Universidad Católica de Chile. Durante 8 semanas, los estudiantes trabajaron tratando de dar solución a los problemas de forma autónoma, con la retroalimentación de sus pares, y de los dos docentes del curso especialistas en Química y en Educación Química.

Al término de la actividad, los estudiantes entregaron un reporte del trabajo desarrollado durante la indagación (bitácora), y presentaron la respuesta a la pregunta formulada inicialmente.

Considerando estos elementos, se solicita a los estudiantes que expliquen el orden de la reacción que estudiaron en su problema. Para ello primero organizan la información teórica y experimental en una $\mathrm{V}$ de Gowin, para facilitar su articulación en la explicación posterior del orden de reacción.

En las explicaciones de los futuros profesores surgen cinco formas de representación del orden de reacción: tabla (A), gráfico (B), ecuación (C), interpretación matemática (D) e interpretación química (E). 
Estudios Pedagógicos XLII, N 4: 243-260, 2016

HACIA LA INTEGRACIÓN DEL CONOCIMIENTO DISCIPLINAR Y PEDAGÓGICO: DESARROLLANDO EL CONOCIMIENTO PEDAGÓGICO DEL CONTENIDO EN LA FORMACIÓN INICIAL DE PROFESORES DE QUÍMICA

Fig. 3: Repertorio de representaciones de la noción de orden de reacción

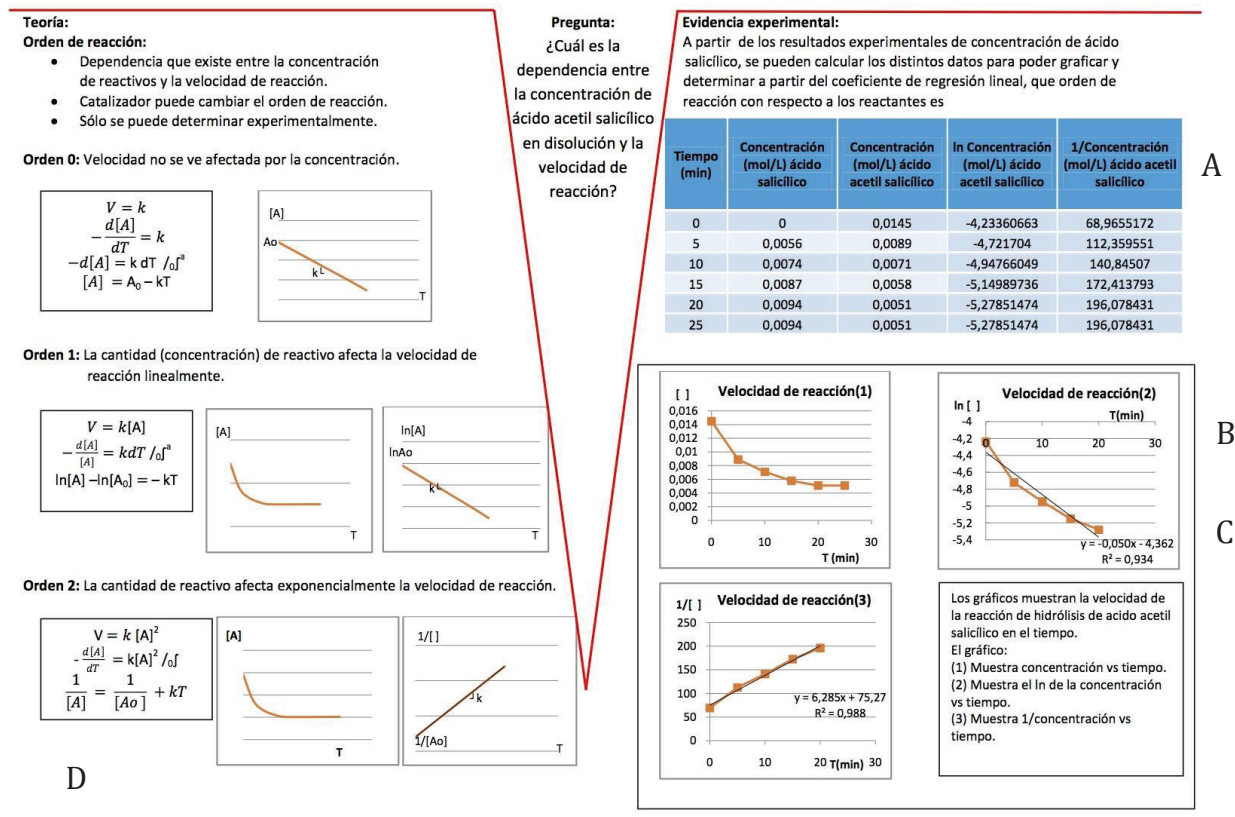

Se observa que el gráfico (3) presentó el coeficiente de regresión lineal más cercano a 1, esto quiere decir, que los datos experimentales obtenidos, se ajustan a este modelo, el cual corresponde a una reacción de orden 2 , esto indica que la cantidad de reactivo afecta de manera exponencial la velocidad de reacción.

El propósito de esta etapa es que los estudiantes activen su conocimiento disciplinar, evalúen la presencia de dificultades en el aprendizaje de la cinética química, y puedan superarlas en el contexto del trabajo desarrollado. Adicionalmente se espera que a través del proceso de indagación, los profesores en formación vayan desarrollando las habilidades propias de la práctica científica.
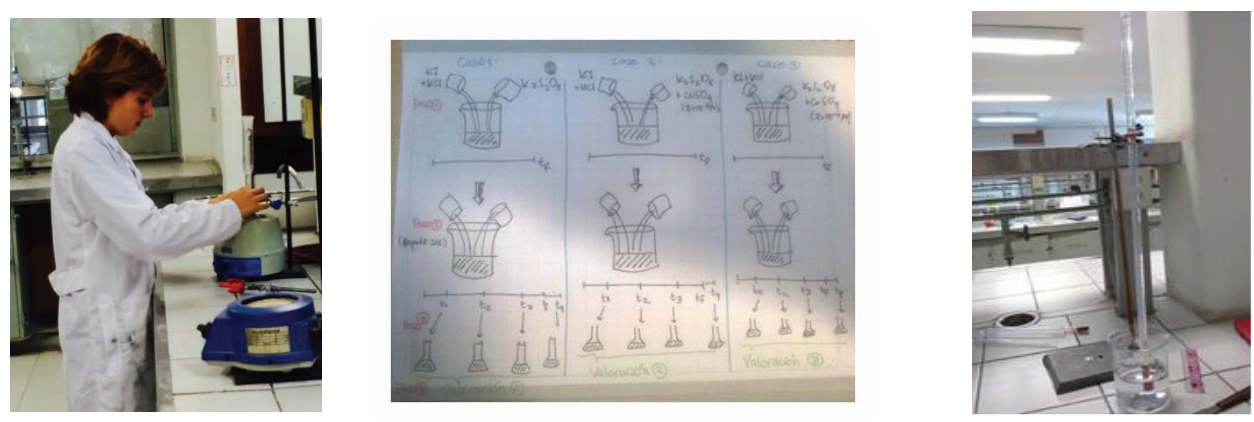


\subsection{PREPARACIÓN Y REPRESENTACIÓN DEL CONTENIDO}

La preparación del contenido involucró la consulta de fuentes documentales consideradas relevantes para la enseñanza de la cinética química: análisis de instrumentos curriculares (planes de estudio y textos escolares) y lectura de resultados de investigación en torno al aprendizaje de la cinética química (Cakmacki, 2010). Esta revisión dio lugar a un esquema conceptual donde se representan las ideas clave que se quiere trabajar y las relaciones temáticas entre ellas, reinterpretando los objetivos de aprendizaje propuestos en el curriculum.

En el contexto de la clase se identifican las diversas

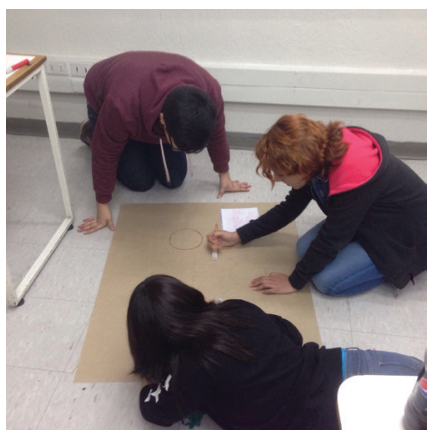
representaciones generadas en las bitácoras, y se discuten sus posibilidades en contextos de enseñanza de la cinética.

En esta etapa se espera que emerjan criterios para la enseñanza del contenido que van más allá de los criterios disciplinarios que se trabajaron en la etapa anterior: criterios curriculares, criterios didácticos y criterios pedagógicos emergen de la discusión en torno a las diversas fuentes documentales consultadas y a las diversas formas de representación de nociones clave como orden de reacción.

\subsection{SELECCIÓN Y ADAPTACIÓN DEL CONTENIDO}

A continuación se les presenta a los profesores en formación el siguiente contexto problemático:

Usted acaba de incorporarse al Instituto Alonso de Ercilla como profesor de Química para Educación Media.

Este instituto es un establecimiento educacional particular pagado de los hermanos maristas, que fue fundado en 1929, atiende a niños y jóvenes de prekinder a $4^{\circ}$ medio, y se ha destacado por su excelencia académica.

En los niveles de Enseñanza Media, hay 4 clases por curso, con una capacidad máxima de 40 estudiantes por clase.

El $3^{\circ}$ medio $C$ es un curso compuesto por 35 estudiantes, que se destacan por ser trabajadores y disciplinados. Sin embargo, algunos profesores se quejan de que son demasiado preocupados de sus notas, poco solidarios y, en general, poco creativos.

Como parte del desarrollo del curso de $3^{\circ}$ medio en este establecimiento educacional, debe abordar la temática: CINÉTICA QUÍMICA, que constituye la tercera unidad del curso. Dentro de esta temática se espera que los estudiantes sean capaces de interpretar, mediante la obtención, organización y procesamiento de información, la velocidad de las reacciones químicas del entorno y su variación en el tiempo (AE8).

Al término de la primera clase, usted ha presentado datos experimentales que corresponden al seguimiento cinético de tres reacciones químicas, mostrando cómo varía la concentración en el tiempo. Al representar gráficamente estos datos, los estudiantes se dan cuenta de que presentan tendencias diferentes, y uno de ellos formula la siguiente pregunta: ¿por qué cuando representamos la concentración cada reacción tiene un gráfico diferente? 
La pregunta del estudiante alude al concepto orden de reacción, y usted se compromete a responder a la pregunta del estudiante en la próxima clase.

Para desarrollar una respuesta apropiada le sugerimos que recolecte todos aquellos criterios que pueden ser relevantes para proponer una explicación que sea apta para ser comprendida por sus estudiantes del $3^{\circ}$ medio $C$ del Instituto Alonso de Ercilla.

Además de los criterios que ya han sido desarrollados en las dos etapas anteriores, se incorporan ahora conocimientos contextuales y de conocimiento de las características de los estudiantes.

Los futuros profesores inician su trabajo consensuando algunos criterios disciplinares a considerar para proponer una respuesta a la pregunta del estudiante. Para ello, organizan los criterios en tres dimensiones: conocimiento químico, conocimiento matemático y habilidades de pensamiento científico. En cada caso, indagan si estas temáticas han sido trabajadas anteriormente de acuerdo al curriculum, y en base a eso, de qué forma podrían abordarlo en la situación problemática que se les presenta, lo que implica incorporar también conocimiento curricular.

La combinación de estos dos tipos de conocimiento da lugar a una primera transformación del conocimiento disciplinar, que debe ajustarse al conocimiento que tienen los estudiantes de educación media en este momento de su formación. A continuación, los profesores en formación proponen algunos lineamientos de cómo abordar las diversas temáticas, incorporando ahora criterios didácticos, aunque son todavía muy poco formales.

En la tabla 1 se presentan los criterios propuestos por uno de los estudiantes:

Tabla 1: Criterios disciplinares, curriculares y didácticos para la transformación de una explicación sobre orden de reacción (Alberto)

\begin{tabular}{|c|c|c|c|}
\hline \multicolumn{2}{|c|}{ Criterios } & $\begin{array}{l}\text { Trabajado o no según lo } \\
\text { declarado en el currículum }\end{array}$ & Cómo abordarlo \\
\hline \multirow{3}{*}{ Conceptos } & Cinética & $\begin{array}{l}\text { Lo están viendo en } \\
\text { tercero medio }\end{array}$ & Breve repaso del concepto. \\
\hline & $\begin{array}{l}\text { Velocidad de } \\
\text { reacción }\end{array}$ & $\begin{array}{l}\text { Lo están viendo en } \\
\text { tercero medio }\end{array}$ & Breve repaso del concepto. \\
\hline & $\begin{array}{l}\text { Orden de } \\
\text { reacción }\end{array}$ & $\begin{array}{l}\text { Lo están viendo en } \\
\text { tercero medio }\end{array}$ & Explicación que hay que desarrollar. \\
\hline \multicolumn{2}{|c|}{ Integrales } & $\begin{array}{l}\text { No es visto hasta } \\
\text { este momento. }\end{array}$ & $\begin{array}{l}\text { Mencionar que existe un concepto } \\
\text { matemático(operación) que permiten ajustar la ley } \\
\text { de velocidad a una ecuación lineal, que finalmente } \\
\text { me permite decidir a qué tipo de grafico se ajustan } \\
\text { mis datos experimentales. }\end{array}$ \\
\hline \multicolumn{2}{|c|}{ Derivadas } & $\begin{array}{l}\text { No es visto hasta } \\
\text { este momento. }\end{array}$ & $\begin{array}{l}\text { Mencionar que existe un concepto } \\
\text { matemático(operación) que permiten ajustar la ley } \\
\text { de velocidad a una ecuación lineal, que finalmente } \\
\text { me permite decidir a qué tipo de grafico se ajustan } \\
\text { mis datos experimentales. }\end{array}$ \\
\hline
\end{tabular}




\begin{tabular}{|c|c|c|c|}
\hline \multicolumn{2}{|c|}{$\begin{array}{l}\text { Coeficiente de regresión } \\
\text { lineal }\end{array}$} & $\begin{array}{l}\text { Se ve el concepto de } \\
\text { desviación estándar en } \\
\text { segundo medio en la } \\
\text { unidad } 4 \text { Datos y azar. }\end{array}$ & $\begin{array}{c}\text { Mencionar que es un método matemático que } \\
\text { modela la relación entre una variable dependiente } \\
\text { y una variable independiente. Si se desea } \\
\text { transformar aún más, es algo similar a la } \\
\text { desviación estándar, pero para ajustar datos } \\
\text { experimentales a una recta. }\end{array}$ \\
\hline \multicolumn{2}{|c|}{ Logaritmo Natural } & $\begin{array}{l}\text { Se debiera mencionar en } \\
\text { segundo medio en la } \\
\text { unidad } 1 \text { Números, en el } \\
\text { AE } 09 \text { o en unidad } 3 \\
\text { Algebra, en el AE } 02 . \\
\end{array}$ & $\begin{array}{l}\text { Mencionar como una función similar a logaritmo } \\
\text { pero que su base no es } 10 \text { sino e. }\end{array}$ \\
\hline \multirow{3}{*}{$\begin{array}{l}\text { Habilidades } \\
\text { previas }\end{array}$} & $\begin{array}{l}\text { Organizar e } \\
\text { interpretar } \\
\text { gráficos/datos }\end{array}$ & $\begin{array}{l}\text { HPC que se trabaja } \\
\text { progresivamente desde } 1^{\circ} \text { a } \\
4^{\circ} \text { medio. Tener en cuenta } \\
\text { que en la evaluación } \\
\text { diagnostica solo } 8\end{array}$ & \multirow{3}{*}{$\begin{array}{c}\text { Será necesario explicar las gráficas mencionando } \\
\text { las variables involucradas, para formular } \\
\text { finalmente la explicación a la pregunta que el } \\
\text { estudiante planteo. }\end{array}$} \\
\hline & $\begin{array}{l}\text { Identificar } \\
\text { Variables }\end{array}$ & $\begin{array}{c}\text { HPC desarrollada en } \\
\text { séptimo básico. Tener en } \\
\text { cuenta la evaluación } \\
\text { diagnostica, en la cual } 13 \\
\text { estudiantes respondieron } \\
\text { incorrectamente las } 2 \\
\text { preguntas que involucraban } \\
\text { esta habilidad. }\end{array}$ & \\
\hline & $\begin{array}{c}\text { Formular } \\
\text { explicaciones }\end{array}$ & $\begin{array}{c}\text { HPC que se trabaja } \\
\text { progresivamente desde } 1^{\circ} \text { a } \\
4^{\circ} \text { medio. Tener en cuenta } \\
\text { que en la evaluación } \\
\text { diagnostica solo } 8 \\
\text { estudiantes obtuvieron la } \\
\text { máxima puntuación. }\end{array}$ & \\
\hline
\end{tabular}

Con los criterios desarrollados, cada uno de los futuros profesores desarrolló su explicación.

Los criterios pedagógicos, de conocimiento del estudiante y contextuales no aparecen en forma explícita, aunque en el diálogo que se establece en la instancia evaluativa final algunos de estos elementos van emergiendo, pero en forma poco estructurada y sin una articulación explícita con el resto de criterios que se han usado.

\subsection{LOS CORES DE LOS FUTUROS DOCENTES AL TÉRMINO DE LA UNIDAD DE CINÉTICA}

Como actividad de término cada uno de los profesores en formación presentó su explicación, y se le formularon las preguntas propuestas por Garritz (2007) para fundamentar las decisiones que tomaron y poder caracterizar el CPC que han desarrollado al término de la actividad. La tabla 2 muestra un resumen de sus respuestas: 


\begin{tabular}{|c|c|c|c|}
\hline \multicolumn{4}{|c|}{$\begin{array}{l}\text { CoRes (Content Representation) } \\
\text { Tema: Cinética química } \\
\text { Contenido específico: Órden de reacción }\end{array}$} \\
\hline Pregunta & Patricia & Laura & Alberto \\
\hline $\begin{array}{llr}\text { (1)¿Qué es lo que } \\
\text { esperas } & & \text { que } \\
\text { aprendan } & & \text { tus } \\
\text { estudiantes } & \text { con } & \text { tu } \\
\text { propuesta? } & & \end{array}$ & $\begin{array}{l}\text { Determinar el orden a } \\
\text { partir de la velocidad de } \\
\text { reacción, mediante } \\
\text { p r o c e d i m i e n t o s } \\
\text { experimentales }\end{array}$ & $\begin{array}{lll}\text { Interpretar el orden } & \text { de } \\
\text { reacción en relación a la } \\
\text { velocidad y y a } & \text { su } \\
\text { representación gráfica. } & \end{array}$ & $\begin{array}{l}\text { Comprender la noción de } \\
\text { orden de reacción y su } \\
\text { determinación a partir de datos } \\
\text { experimentales a los que se } \\
\text { aplican operaciones } \\
\text { matemáticas, y diferenciarlo } \\
\text { delcoeficiente estequiométrico. }\end{array}$ \\
\hline $\begin{array}{l}\text { ¿Por qué te } \\
\text { parece que es } \\
\text { importante que los } \\
\text { estudiantes sepan lo } \\
\text { que es el orden de } \\
\text { reacción? }\end{array}$ & $\begin{array}{l}\text { Promueve la } \\
\text { interpretación de gráficos } \\
\text { Ayuda a comprender las } \\
\text { reacciones y sus distintas } \\
\text { velocidades } \\
\text { Ayuda a comprender lo } \\
\text { que trabajarán } \\
\text { posteriormente de } \\
\text { cinética, y su relevancia }\end{array}$ & $\begin{array}{l}\text { Se enfoca en la velocidad, } \\
\text { que es un concepto que la } \\
\text { gente no asocia a este punto } \\
\text { de vista. } \\
\text { Agrega la noción de } \\
\text { velocidad a todas las } \\
\text { reacciones químicas. } \\
\text { Promueve la identificación } \\
\text { de variables y r la } \\
\text { interpretación de la la } \\
\text { dependencia entre ellas }\end{array}$ & $\begin{array}{l}\text { Comprender que los } \\
\text { fenómenos químicos que se } \\
\text { dan cotidianamente no } \\
\text { proceden de la misma forma ni } \\
\text { a la misma velocidad, y qué } \\
\text { factores los afectan }\end{array}$ \\
\hline $\begin{array}{l}\text { (3) ¿Qué saben los } \\
\text { estudiantes en torno } \\
\text { a esta idea y de qué } \\
\text { forma puede afectar } \\
\text { al aprendizaje? }\end{array}$ & $\begin{array}{l}\text { Ya han visto cinética y } \\
\text { velocidad de reacción, } \\
\text { conceptos necesarios } \\
\text { para entender el orden de } \\
\text { reacción. } \\
\text { Conocen la noción de } \\
\text { desviación estándar, pero } \\
\text { probablemente de forma } \\
\text { vaga. }\end{array}$ & $\begin{array}{l}\text { Ya han visto cinética, } \\
\text { velocidad yu } \\
\text { representación. } \\
\text { No han visto derivadas e } \\
\text { integrales, sería muy } \\
\text { complejo trabajarlo, y no es } \\
\text { un requisito. }\end{array}$ & $\begin{array}{l}\text { Ya han visto cinética y } \\
\text { velocidad de reacción, pero es } \\
\text { igualmente importante } \\
\text { revisarlos. } \\
\text { Deberán aplicar el coeficiente } \\
\text { de regresión lineal para } \\
\text { comparar las distintas gráficas, } \\
\text { como parámetro matemático } \\
\text { que modela la dependencia } \\
\text { entre variables. También es } \\
\text { necesario que apliquen } \\
\text { entonces la idea de variables y } \\
\text { su relación, junto con la } \\
\text { interpretación de gráficas. }\end{array}$ \\
\hline $\begin{array}{l}\text { (4) ¿qué es lo que } \\
\text { crees que les va a } \\
\text { costar más a los } \\
\text { estudiantes de esta } \\
\text { explicación que les } \\
\text { estas dando? }\end{array}$ & $\begin{array}{l}\text { La falta de base } \\
\text { matemática hace que no } \\
\text { puedan comprender las } \\
\text { expresiones matemáticas } \\
\text { que se usan para } \\
\text { identificar el orden de } \\
\text { reacción. }\end{array}$ & $\begin{array}{l}\text { Las ecuaciones y cómo } \\
\text { llegar a esta representación } \\
\text { me causaría un problema, } \\
\text { por lo que me centraría en } \\
\text { entender que hay diversas } \\
\text { representaciones. }\end{array}$ & $\begin{array}{l}\text { El ajuste de los datos va a } \\
\text { requerir una explicación más } \\
\text { detallada, y cómo enfrentar a la } \\
\text { pregunta de por qué se } \\
\text { producen distintas } \\
\text { dependencias en la velocidad } \\
\text { de reacción }\end{array}$ \\
\hline $\begin{array}{l}\text { ¿qué identificas tú } \\
\text { que has aprendido? }\end{array}$ & $\begin{array}{l}\text { Ahora sabría como } \\
\text { enfrentar un problema } \\
\text { químico. } \\
\text { Adaptar la explicación } \\
\text { para una sala de clase fue } \\
\text { un desafío súper grande, } \\
\text { no es obvio como } \\
\text { hacerlo. }\end{array}$ & $\begin{array}{l}\text { Me di cuenta de que las } \\
\text { cosas no son } \\
\text { necesariamente como me } \\
\text { las enseñaron, y quizás el } \\
\text { mejor camino no es el que } \\
\text { yo conozco. } \\
\text { Ha sido un proceso de } \\
\text { constante reflexión, me he } \\
\text { sentido complicada, } \\
\text { desafiada. Pero en las } \\
\text { discusiones con mis pares } \\
\text { siento que voy avanzando, } \\
\text { y que ya estoy pensando de } \\
\text { otra manera, más completa } \\
\text { y teniendo en cuenta otros } \\
\text { factores. Ahora me imagino } \\
\text { haciendo clase de una } \\
\text { forma totalmente distinta. }\end{array}$ & $\begin{array}{l}\text { Empiezo a visualizar la } \\
\text { posibilidad de experimentar en } \\
\text { un contexto escolar, y acercar } \\
\text { la Química a la vida cotidiana } \\
\text { de las personas. } \\
\text { He repasado conceptos que ya } \\
\text { sabía, y los he entendido mejor, } \\
\text { más allá de los procedimientos } \\
\text { matemáticos. Me di cuenta de } \\
\text { que yo mismo no tenía clara la } \\
\text { diferencia entre orden y } \\
\text { coeficiente estequiométrico. } \\
\text { Creo que he avanzado en } \\
\text { cuanto a mis propias } \\
\text { habilidades de pensamiento } \\
\text { científico. } \\
\text { Ahora estoy aprendiendo lo } \\
\text { que realmente necesito saber } \\
\text { para enseñar. }\end{array}$ \\
\hline
\end{tabular}




\section{REFLEXIÓN FINAL}

El diseño de las actividades para el desarrollo del CPC se hizo en base a los antecedentes de la literatura, y en la medida que las hemos ido implementando, hemos ido delimitando mejor este tipo de conocimiento y qué tipo de actividades pueden ser efectivas para promoverlo.

En los primeros CoRes de estos profesores en formación, la integración del conocimiento pedagógico y disciplinar es todavía incipiente, cada uno muestra diversos criterios que aplica a la construcción de una explicación, de forma poco articulada y sin una base teórica de los cursos precedentes. A falta de experiencia práctica, los futuros profesores acuden al curriculum como referencia de lo que el estudiante puede saber, y sus nociones de conocimiento precedente se centran en el conocimiento escolar, más que en el conocimiento cotidiano que sus estudiantes puedan tener.

Sin embargo, vemos algunos indicios prometedores en esta primera experiencia: los profesores en formación perciben que la secuencia de aprendizaje es un desafío que provoca una ruptura con sus creencias sobre la enseñanza, lo que sería un requisito para una buena formación docente (Furió, 1994). Los futuros profesores empiezan a plantearse nuevas preguntas, que les llevan a una reelaboración de su conocimiento para enseñar: en su visión de la enseñanza aparecen nuevos factores, todavía vagos y poco organizados. A través de las instancias que se van proponiendo, se van abriendo espacios de reflexión donde el conocimiento incidental de la enseñanza, los aprendizajes de los cursos disciplinares, pedagógicos y didácticos, y las experiencias en el contexto de sus prácticas se van haciendo explícitos en respuesta a las demandas que el curso les plantea. Como formadores de formadores, nuestro rol es gestionar sus respuestas y organizar las ideas en estructuras coherentes, darles mayor fundamentación teórica y visibilizar nuestra propia forma de pensar en la enseñanza de determinados contenidos, nuestro propio CPC. El propósito es que sean cada vez más autónomos en su forma de generar representaciones del contenido químico y seleccionar las que son más adecuadas de acuerdo a criterios pedagógicos, didácticos, disciplinares, curriculares, contextuales, explícitos y fundamentados en su conocimiento teórico y práctico.

Empezar a generar oportunidades de articular el conocimiento disciplinar y pedagógico, en el contexto de la formación inicial de profesores, permite formalizar el desarrollo del conocimiento pedagógico del contenido. Esto les permite a profesores que todavía no empiezan su ejercicio profesional hacer explícitos sus criterios para la enseñanza, y poder incorporar otros elementos que emergen en el trabajo con recursos diversos (curriculum, literatura especializada, etc.) con el andamiaje de especialistas disciplinares y didácticos. Además, este proceso se enriquece con la interacción con sus pares, en las diversas instancias de discusión que se proponen en el curso.

Estamos convencidos que este tipo de trabajo, que puede enmarcarse en un modelo de formación inicial de profesores paralelo simultáneo, involucra procesos que requieren de un enfoque reflexivo -y no instructivo- para que realmente contribuyan al desarrollo profesional docente, desarrollando las competencias profesionales que deberán permitir a los profesores continuar con estos procesos en forma autónoma en su ejercicio profesional.

\section{REFERENCIAS BIBLIOGRÁFICAS}

Abell, S. (2007). Research on science teacher knowledge. en S. Abell \& N. Lederman (Eds.), Handbook of research on science education (pp. 1105-1149). Mahwah, NJ: Lawrence Erlbaum. 
Abell, S. (2008). Twenty years later: does pedagogical content knowledge remain a useful idea? International Journal of Science Education, vol.30, n.10, 1405-1416.

Acevedo, J. A. (2009). Conocimiento didáctico del contenido para la enseñanza de la naturaleza de la ciencia (I): el marco teórico. Eureka, 6(1), 21-46.

Ávalos, B. (2005). Las instituciones formadoras de docentes y las claves para formar buenos docentes. En R. Lara \& D. Rojas (Comp.), El desafio de formar los mejores maestros: situación actual, experiencias, innovaciones y retos en la formación de los formadores docente (pp. 1422). Bogotá: Universidad Pedagógica Nacional.

Ball, D. \& Forzani, F. (2009). The Work of Teaching and the Challenge for Teacher Education. Journal of Teacher Education, 60(5), 497-511.

Barber, M., \& Mourshed, M. (2008). Cómo hicieron los sistemas educativos con mejor desempeño para alcanzar sus objetivos. PREAL.

Bolívar, A. (2007). La formación del profesorado: entre la posibilidad y la realidad. En RomeroMorante, J. y Luis-Gómez, A. (Eds.), La formación del profesorado a la luz de una "profesionalidad democrática" (pp. 79-120). Santander: Consejería de Educación de Cantabria.

Borowski, A., Carlson, J., Fischer, H.E., Henze, I., Gess-Newsome, J., Kirschner S., \& Van Driel, J.H. (2012). Different models and methods to measure teachers pedagogical content knowledge. Paper presentado en la reunión anual de la National Association for Research in Science Teaching (NARST), Indianapolis.

Cakmakci, G. (2010). Identifying Alternative Conceptions of Chemical Kinetics among Secondary School and Undergraduate Students in Turkey. J. Chem. Educ., 87 (4), 449-455.

Camacho, J. P., Jiménez, J., Galaz J. A. y Santibáñez, D. (2010). La Formación de profesores de Ciencia en el mundo: una revisión. En: Cofré, H. (ed.) Cómo mejorar la enseñanza de las ciencias en Chile. (pp. 19-40). Santiago de Chile: Ediciones UCSH.

Carrascosa, J., Martínez, J., Furió, C. y Guisasola, J. (2008). ¿Qué hacer en la formación inicial del profesorado de ciencias de secundaria? Rev. Eureka Enseñ. Divul. Cien., 5, 118-133.

Chung Wei, R., Darling-Hammond, L., Andree, A., Richardson, N., and Orphanos, S. (2010). Professional Learning in the Learning Profession. A Status Report on Teacher Development in the U.S. and Abroad. Technical Report. School Redesign Network, Stanford University.

Cofré, H., Camacho, J., Galaz, A., Jiménez, J., Santibáñez, D. y Vergara, C. (2010). La educación científica en Chile: debilidades de la enseñanza y futuros desafíos de la educación de profesores de ciencia. Estudios Pedagógicos, vol.26, n.2, 279-293.

Cofré, H. \& Vergara, C. (2010) La formación de profesores de ciencia en Chile: desarrollo, estado actual y futuros desafíos. En Cofré, H. (ed.) Cómo mejorar la enseñanza de las ciencias en Chile (pp 257-278). Santiago: Ediciones Universidad Católica Silva Henríquez.

Esteve, J.M. (2003). La tercera revolución educativa. La educación en la sociedad del conocimiento. Barcelona: Paidós.

Furió, C., (1994). Tendencias actuales en la formación del profesorado de ciencias, Enseñanza de las Ciencias, 12(2) pp. 188-199.

Furió, C. (1995). Preconcepciones del profesorado de Ciencias sobre algunos aspectos del proceso de enseñanza-aprendizaje. A Distancia, otoño, 57-61.

Garritz, A. (2007). Análisis del conocimiento pedagógico del curso "ciencia y sociedad" a nivel universitario. Eureka, 4(2), 226-246.

Harlen, W. (2010). Principles and Big Ideas of Science Education. Hatfield, Herts: Association for Science Education.

Kind, V. (2009). Pedagogical content knowledge in science education: perspectives and potential for progress. Studies in Science Education, 45(2), 169-204.

Lee, E y Luft, J. A. (2008). Experienced secondary science teachers' representation of pedagogical content knowledge. International Journal of Science Education, 30(10), 1343-1363.

Loughran, J.J., Mulhall, P., \& Berry, A. (2004). In search of pedagogical content knowledge in 
HACIA LA INTEGRACIÓN DEL CONOCIMIENTO DISCIPLINAR Y PEDAGÓGICO: DESARROLLANDO EL

CONOCIMIENTO PEDAGÓGICO DEL CONTENIDO EN LA FORMACIÓN INICIAL DE PROFESORES DE QUÍMICA

science: Developing ways of articulating and documenting professional practice. Journal of Research in Science Teaching, 41(5), 370-391.

Loughran, J. J., Berry, A. y Mulhall, P. (2006). Understanding and developing science teachers' pedagogical content knowledge. Rotterdam: Sense Publishers.

Loughran, J.J., Berry, A. y Mulhall, P. (2012). Understanding and developing science teachers' pedagogical content knowledge. 2a edición. Rotterdam: Sense Publishers.

Ministerio de Educación [MINEDUC]. (2012). Estándares orientadores para carreras de pedagogía en educación media. Santiago de Chile: CIAE-CEPPE.

Minner, D. D., Levy, A. J., and Century, J. R. (2009). Inquiry-based science instruction-what is it and does it matter? Results from a research synthesis years 1984-2002. J. Res. Sci. Teach, 1-24.

Park, S., \& Oliver, J. (2008). Revisiting the conceptualization of pedagogical content knowledge (PCK): PCK as a conceptual tool to understand teachers as professionals. Research Science Education, 38(2), 261-284.

Schmelzing, S., Van Driel, J. H., Jüttner, M., Brandenbusch, S., Sandmann, A. \& Neuhaus, B. (2013). Development, evaluation, and validation of a paper-and-pencil test for measuring two components of biology teachers' pedagogical content knowledge concerning the "cardiovascular system”. International Journal of Science and Mathematics Education, 11(6), 1369-1390.

Shulman, L.S. (1987). Knowledge and teaching: foundations of the new reform. Harvard Educational Review, 57(1), 1-22.

Shulman, L. (2005). Conocimiento y enseñanza: fundamentos de la nueva reforma. Profesorado. Revista de curriculum y formación del profesorado, 9(2), 1-30.

Talanquer, V. (2004). ¿Qué conocimiento distingue a los buenos maestros de química? Educación Química, 15(1), 52-57.

Vergara, C., \& Cofré, H. (2014). Conocimiento Pedagógico del Contenido: ¿el paradigma perdido en la formación inicial y continua de profesores en Chile?. Estudios pedagógicos, XL, nº especial $1,323-338$. 